\title{
Design and Fabrication of a Thin-Film Silicon Photodetector with Angular Sensitivity for Optical Micro-Encoder
}

\author{
Bongseok Choi Non-member (Tohoku Univerisity, choi@hane.mech.tohoku.ac.jp) \\ Yoshiaki Kanamori Member (Tohoku Univerisity, kanamori@hane.mech.tohoku.ac.jp) \\ Kazuhiro Hane Member (Tohoku Univerisity, hane@hane.mech.tohoku.ac.jp)
}

Keywords : Thin-film photodetector, directional sensitivity, optical absorbance, resonant grating, subwavelength grating

In this paper, we describe a directional thin-film silicon photodetector with the objective of optical micro-encoder application. A directional photodetector with high absorption efficiency at a specific angle of incident light is based on guided-mode resonance (GMR) of subwavelength grating. The sensitive direction is tunable by changing the grating period. Numerical analysis, fabrication and device performance are presented.

The optical absorbance in the thin silicon layer is calculated for various grating periods. Rigorous coupled-wave analysis (RCWA) is utilized in the calculation for TE-polarized incident light at 783 $\mathrm{nm}$ incident wavelength. The calculated results are shown in fig. 1(a). The optical absorbance in also calculated by RCWA for the case without resonant grating as shown in fig. 1(b). When the grating is formed on the top silicon layer, the calculated absorbance is higher than 0.8 for the grating period $\Lambda$ from 330 $\mathrm{nm}$ to $420 \mathrm{~nm}$. In the case of top silicon layer without the resonant grating, the absorbance is smaller than $5 \%$. When the grating period $\Lambda$ changed from $310 \mathrm{~nm}$ to $450 \mathrm{~nm}$ in the proposed structures, sensitive angle also changes from 0 degrees to 50 degrees with the high absorbance characteristics. Moreover, it is noted that the angular sensitivity can be clearly tuned by changing the grating period.

Figure 2 shows the optical micrograph of the fabricated photodetector. The grating area covers $500 \times 650 \mu \mathrm{m}^{2}$ of the active $1000 \times 1500 \mu \mathrm{m}^{2}$ photodetector region. Due to the subwavelength grating period, a low reflection was achieved in the region.

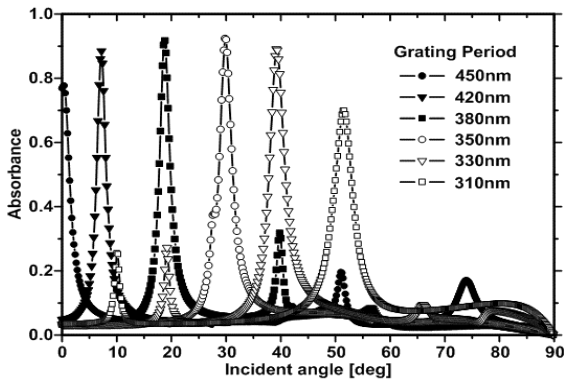

(a)

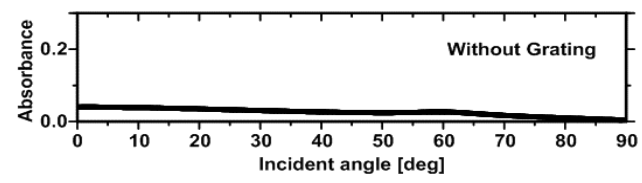

(b)

Fig. 1. Directional absorbance characteristics versus resonant grating period. (a) with resonant grating (b) without resonant grating
As depicted in fig. 3, the directional sensitivities were measured at the reverse bias voltage of $3 \mathrm{~V}$ for the $500 \times 650 \mu \mathrm{m}^{2}$ active resonant grating area with the periods of $450 \mathrm{~nm}$ and $350 \mathrm{~nm}$ at the wavelength of $783 \mathrm{~nm}, 6 \mathrm{~mW}$ incident light under TE polarization. The photocurrents measured as a function of the incident angle clearly show the resonant responses at the angle of 0 degrees and 25 degrees for the $450 \mathrm{~nm}$ and $350 \mathrm{~nm}$ periods, respectively. The directional angle for $350 \mathrm{~nm}$ period grating was about 25 degrees, which was smaller than that calculated (30 degrees) because of the fabrication errors. The directional sensitivity can be tuned by the change of the grating period. In the case of $450 \mathrm{~nm}$ grating period, the resonant response is obtained at the angle of 0 degrees, which agrees well with that obtained in the theoretical calculation. The enhanced photocurrents are less than the values expected from the absorbance shown in fig. 1 . It can be considered the indistinct relations between the absorbed light and the photocurrents from the photoelectric effects, and imperfect boundary conditions or light scattering in the fabricated grating and layers are significant limiting the quantitative comparison between the measurement and calculation. However, the resonant phenomena in the thin silicon layer have been confirmed as shown in fig. 3.

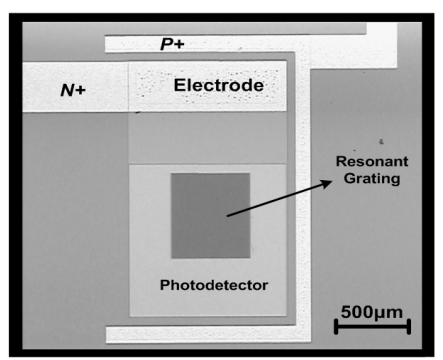

Fig. 2. Micrographs of the fabricated directional thin-film photodetector

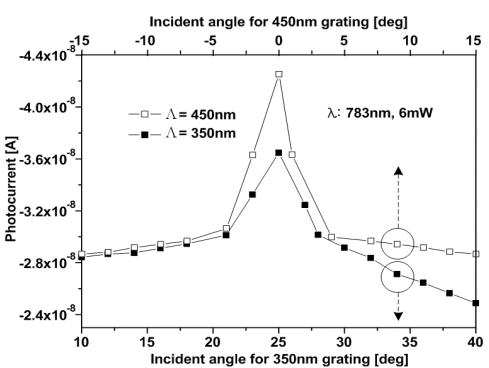

Fig. 3. Measured directional responsivity in the fabricated thin-film photodetector for $350 \mathrm{~nm}$ and 450 $\mathrm{nm}$ period gratings 


\title{
Design and Fabrication of a Thin-Film Silicon Photodetector with Angular Sensitivity for Optical Micro-Encoder
}

\author{
Bongseok Choi* Non-member \\ Yoshiaki Kanamori* Member \\ Kazuhiro Hane* Member
}

In this paper, we describe a directional thin-film silicon photodetector with the objective of optical micro-encoder application. A directional photodetector with high absorption efficiency at a specific angle of incident light is based on guided-mode resonance (GMR) of subwavelength grating. The sensitive direction is tunable by changing the grating period. Numerical analysis, fabrication and device performance are presented.

Keywords : Thin-film photodetector, directional sensitivity, optical absorbance, resonant grating, subwavelength grating.

\section{Introduction}

In opto-mechatronic systems, optical encoders are indispensable sensing components for precise position and displacement measurements. Conventional Moire-type encoders are being utilized in the linear displacement sensing, since the light intensity of Moire fringes are sensitive to the relative lateral displacements between scale and index gratings, and the optical configuration is very simple. As a similar method, a grating-image type encoder with transmission grating was also proposed to compose an integrated and miniaturized micro-encoder ${ }^{(1)}$. However sensor performances based on Moire Effect are still affected by the harmonic noise and signal contrast even when the air gaps between the two gratings remain unchanged. The signal distortion is caused due to the diffraction and interference among $0, \pm 1$ and higher order light beams transmitted through the scale grating.

An interferometric type encoder was also proposed to achieve high sensing resolution ${ }^{(2)}$. Although efficient detection is achieved via the interference of two diffracted beams from scale gratings, these interferometric sensors require several additional optical components and high technical alignment for an acceptable output quality.

Lately, a monolithic optical phase-shift detector on silicon substrate was presented $^{(3)}$. Displacement sensing was experimentally validated via phase-difference between two coherent beams. In the detector, the index grating combined directly with a silicon $p n$-junction is used as a mixing element of the two coherent beams.

Photodetectors aiming optical sensing in particular, Sasaki et al., for instance, used subwavelength-scale grating silicon photodetectors to miniaturize inteferometric sensors ${ }^{(4)}$. Moreover, in order to improve the optical coupling efficiency and responsivity in silicon-on-insulator (SOI) based photodetectors, several groups have followed such approaches as the use of metal-semiconductor-metal structures, surface plasmon resonance effects $^{(5)-(8)}$, etc. Waveguide gratings have been studied for input/output coupling in silicon waveguides ${ }^{(9)}$. Multi-layered

* Department of Nanomechanics, Tohoku University

Aza Aoba 6-6-01, Aramaki, Aoba-ku, Sendai 980-8579 structures combined with various modulated resonant gratings have been used for filtering, wavelength division and spectral sensing ${ }^{(10)}$. Especially, guided-mode resonance (GMR) structures consisting of subwavelength gratings and thin-film layers have been intensively studied in optical filter applications ${ }^{(11)}$. GMR structures exhibit excellent properties such as high efficiency, narrow spectral band, and large bandwidth.

In this paper, a directional thin-film photodetector with angular sensitivity to coherent incident light is demonstrated. The proposed photodetector can be used as a phase-shift detector ${ }^{(3)}$ with a high sensitivity expected from a GMR structure. In the case of Moire-type configuration, sensitive direction of photodetector is designed to be equal to that of \pm 1 order diffracted lights of the scale grating. The encoder signal generated by \pm 1 order diffracted light can be effectively detected even though the zero-order diffracted light exists. Therefore, an encoder with a very small grating period and less-sensitivity to zero-order diffracted light can be accomplished, which suppresses the signal change due to the gaps and harmonics. Here, the design and fabrication of the proposed photodetector are reported.

\section{Sensor Structure and Principle}

The proposed encoder is based on phase-shift detection using phase difference of two coherent incident beams ${ }^{(3)}$. The proposed photodetector working as index grating is placed directly behind the scale grating instead of two-beam separation configuration used in the interferometric encoder ${ }^{(2)}$, because of the better directional characteristics of the photodetector as aforementioned. The schematic diagram of the proposed optical encoder is shown in fig. 1. The sensor consists of a main scale grating and angle-sensitive thin-film photodetector, which performs a key function in the proposed encoder. Although photodetectors and index gratings are usually separated in conventional bulky encoders, in this study, they are monolithically integrated on the same substrate.

The coherent beam illuminates the scale grating. This beam is separated into the transmitted beams in the diffraction orders 0 , \pm 1 and higher. Diffracted beams then directly impinge on the photodetector at the respective angles. The light beams 


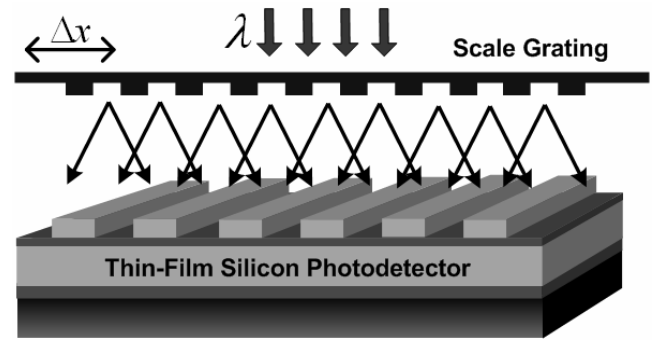

(a)

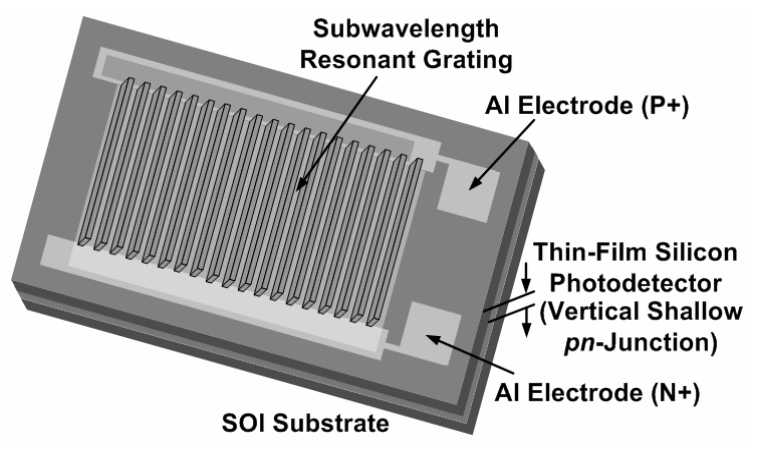

(b)

Fig. 1. Schematic of the proposed encoder by using directional photodetector. (a) encoder driving diagram (b) conceptual structure of the photodetector.

propagating into the top silicon layer through the index grating interfere each other to generate photocurrent. When the scale grating with the pitch, $P$ is moved along $x$ direction with a displacement of $\Delta x$, phase-shifts $s_{ \pm}$of the two \pm 1 order diffraction beams transmitted into the top silicon layer occur, and their value, respectively, can be expressed as;

$$
s_{ \pm}= \pm 2 \pi \times \Delta x / P
$$

The resultant intensity of the encoder, $I_{\mathrm{E}}$, is derived by the conjugated electric fields of the two beams and is expressed by

$$
I_{E} \propto(1+\cos (2 k z))\left(1+\cos \left(s_{+}-s_{-}\right)\right)
$$

where $k$ is the wave number, $z$ is the vector of beam direction to the photodetector.

Figure 2 shows the cross-sectional structure of the designed angle-sensitive thin-film photodetector. The proposed device is based on an SOI substrate. The SOI wafer consists of a $380 \mathrm{~nm}$ -thick top active silicon layer with an $1 \mu \mathrm{m}$-thick buried oxide layer on a silicon substrate. A shallow $p n$-junction is formed vertically in active silicon layer to detect the photocurrent. Rectangular resonant grating with a subwavelength period is made of a $100 \mathrm{~nm}$-thick $\mathrm{Ta}_{2} \mathrm{O}_{5}$ dielectric material (refractive index, $\mathrm{n}=2.1$ ) on a $50 \mathrm{~nm}$-thick $\mathrm{SiO}_{2}$ layer. The typical GMR structure consists of a rectangular subwavelength grating on a high refractive index slab waveguide (the top silicon layer), which has the same configuration as the waveguide grating coupler.

When subwavelength gratings are illuminated with an oblique incident light beam, most of the light propagates directly through the top silicon layer under non-resonant condition. The remaining light is diffracted by the grating into the top silicon layer, which has the high refractive index slab, and it is partially diffracted outwards both in the transmission and reflection directions.

In the proposed device, at a specific incident angle between the grating structure and the incident light, and at a specific

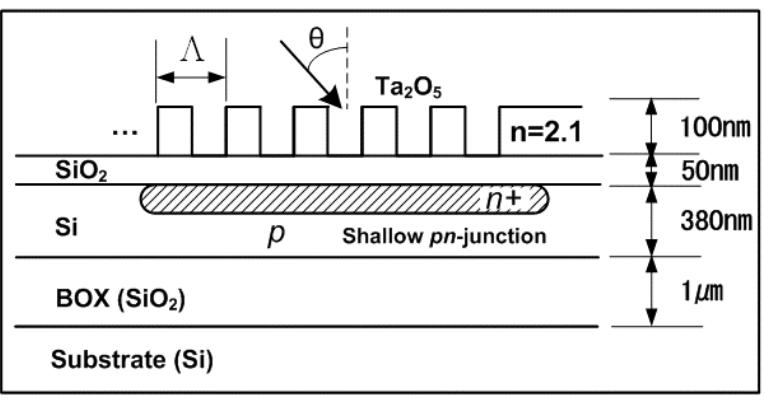

Fig. 2. Cross-sectional structure of the directional thin-film photodetector.

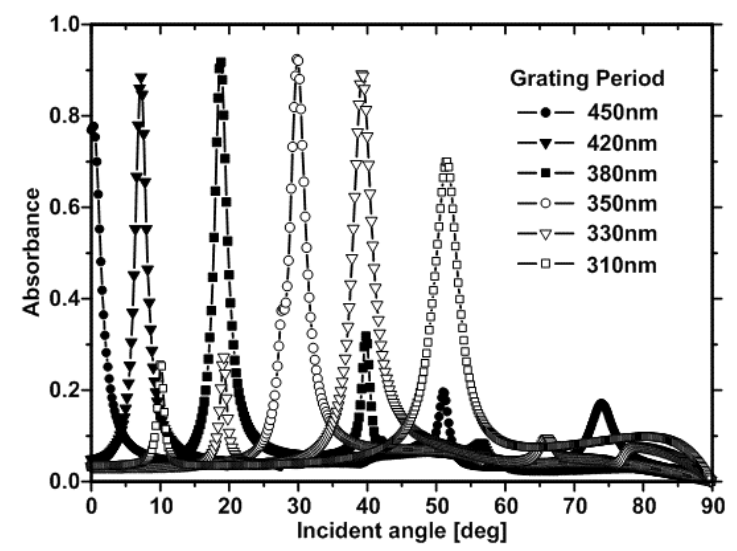

(a)

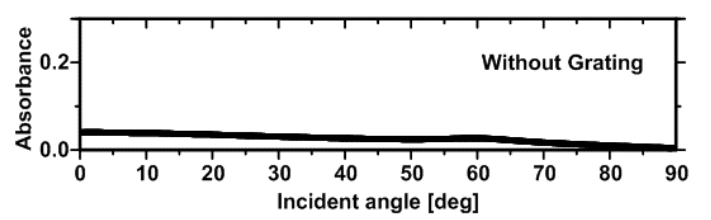

(b)

Fig. 3. Directional absorbance characteristics versus resonant grating period. (a) with resonant grating (b) without resonant grating.

wavelength, a resonant condition (GMR) occurs, where the reflection and transmission values decrease significantly. Thus, the optical absorption is maximized, and the waveguided light is detected efficiently in the silicon layer. Most of the light is confined in the top silicon layer, and then enhanced photocurrent is generated by the interfering light multiply reflected and diffracted by the resonant grating.

The optical absorbance in the thin silicon layer is calculated for various grating periods. Rigorous coupled-wave analysis (RCWA) is utilized in the calculation for TE-polarized incident light. The grating is assumed to be laterally infinite extent. The calculated results are illustrated in fig. 3(a) as a function of incident angle. The wavelength of the incident light is $783 \mathrm{~nm}$. Silicon exhibits low optical absorption characteristic due to its indirect band gap, thus requiring long absorption path. The light intensity along the depth can be expressed in the exponential form, when the silicon surface is illuminated with the light, such that

$$
I=I_{0} e^{-\alpha w}
$$

where $I$ is the optical intensity, $\alpha$ is the absorption coefficient of silicon, and $w$ is the depth from silicon surface. Thus, the optical 
absorbance is about $4 \%$ at the wavelength of $783 \mathrm{~nm}$ along the thickness of the thin-film silicon layer. Similarly, optical absorbance is also calculated by RCWA for the sensor without the resonant grating. The result is shown in fig. 3(b) as a function of the incident angle. When the grating is formed on the top silicon layer, the absorbance is calculated to be higher than 0.8 for the grating period $\Lambda$ from $330 \mathrm{~nm}$ to $420 \mathrm{~nm}$. In the case of top silicon layer without the resonant grating, the absorbance is smaller than $5 \%$. When the grating period $\Lambda$ changed from $310 \mathrm{~nm}$ to $450 \mathrm{~nm}$ in the proposed structures, sensitive angle also changes from 0 degrees to 50 degrees with the high absorbance characteristics. Moreover, it is noted that the angular sensitivity can be clearly tuned by changing the grating period.

Figure 4 shows the numerical analysis results of the reflectance, transmittance and absorbance, in TE polarization for $350 \mathrm{~nm}$ grating period as a function of the incident angle. Although the top silicon layer is thinner than the incident light wavelength, nearly $90 \%$ of the incident light is absorbed at 30 degrees through the

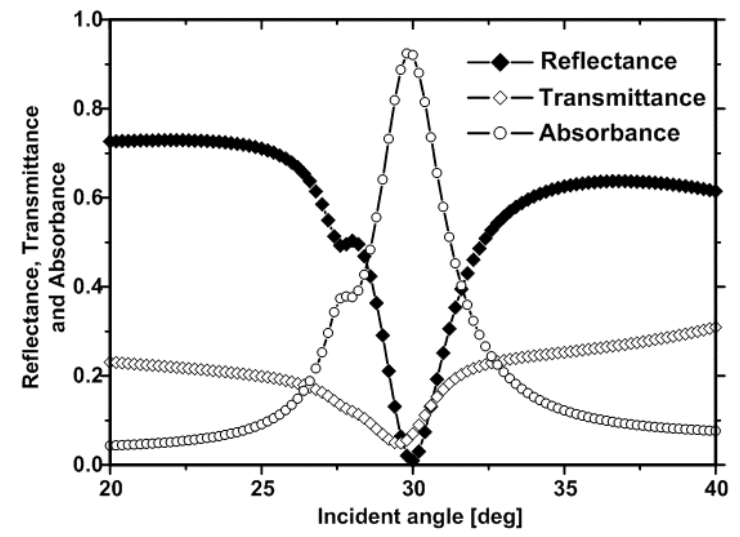

Fig. 4. Numerically calculated reflectance, transmittance and absorbance in TE polarization for $350 \mathrm{~nm}$ grating period.

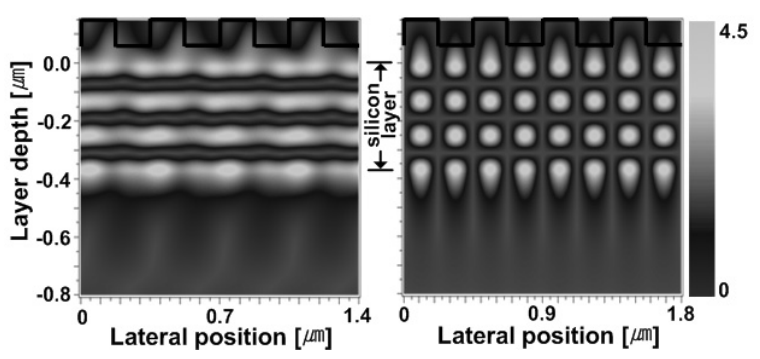

(a)

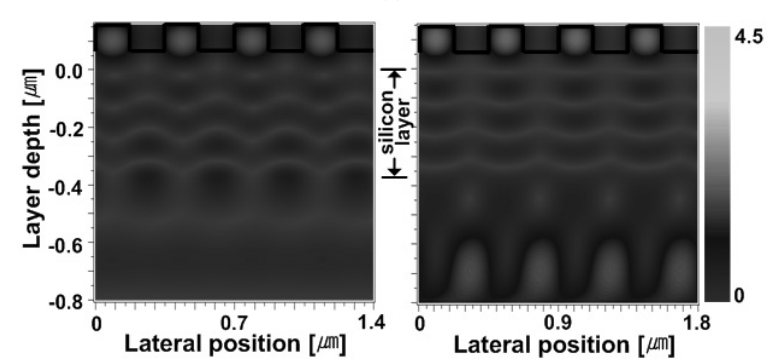

(b)

Fig. 5. Electric field amplitude distributions in the photodetector layer (a) resonant conditions (b) non-resonant conditions for $350 \mathrm{~nm}$ (left) and $450 \mathrm{~nm}$ (right) grating periods.

\section{GMR behavior.}

Although the reflectance is higher than 0.5 at the non-resonant conditions such as 20 25degrees and 35 40degrees ranges in fig. 4 , the encoder, in this study, is used at the resonant condition (for the incident angle at the minimum reflectance ie, 30 degrees). In the optical configuration for the encoder, we set the \pm 1 order diffraction angle to the resonant angle using a proper scale grating. Alghough the zero-order diffraction light may influence a little on the encoder signal, it is expected to the small due to the non-resonant condition as shown in fig. 3 .

Figure 5(a) shows the electric field distributions at the angles of 30 degrees and 0 degrees for single beam incidence in the cases of $350 \mathrm{~nm}$ and $450 \mathrm{~nm}$ period grating, respectively. The enhanced peak intensities are localized within the top silicon layer at the resonant incident angle, proving the fact that the enhanced photocurrent is achievable. In contrast to it, low amplitudes are localized under the non-resonant condition for the incident angle as shown in fig. 5(b). In this non-resonant conditions (for $\Lambda=350$ $\mathrm{nm}, 20$ degrees of incident angle, $\lambda=783 \mathrm{~nm}$ ), the value of maximum amplitude calculated in the top silicon layer is just 0.8 , which is 5.6 times smaller compared to the maximum value of 4.5 in the case of resonant conditions.

\section{Fabrication}

Silicon micromachining and conventional complementary metal-oxide semiconductor (CMOS) compatible processes are employed in the device fabrication. The process flow is schematically illustrated in fig. 6 . The $p n$-junction is formed by carrying out passivation, metallization and dielectric grating fabrication in the top silicon layer. In such a thin-film silicon layer, $p n$-junction is often formed in the lateral direction to the silicon substrate, because of difficulty in the use of conventional bulk photodetector formation process. In this study, however, we designed a $p n$-junction in the vertical direction to form the effective depletion region using a low-energy ion implantation and rapid thermal annealing (RTA) process.

The devices were fabricated on a $14-22 \Omega \cdot \mathrm{cm}$ p-type (100)-oriented SOI wafer (a). The SOI substrate consists of a 500

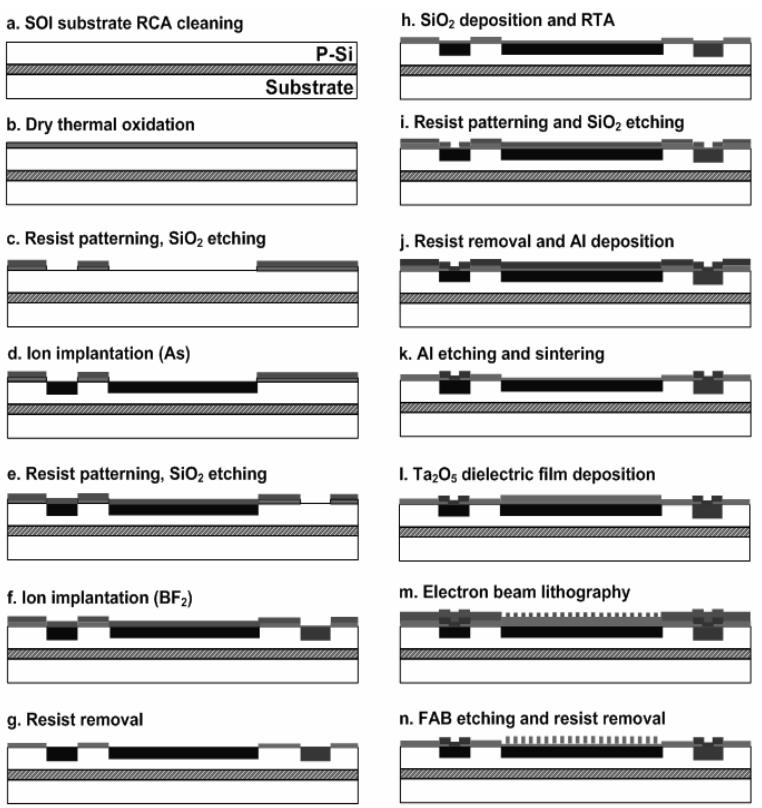

Fig. 6. Process flow of the fabricated device. 
nm-thick top active silicon layer on a $1 \mu \mathrm{m}$-thick buried oxide layer. Silicon substrate is $670 \mu \mathrm{m}$-thick. The top silicon layer is reduced to $380 \mathrm{~nm}$ thick by thermal oxidation in furnace and a $\mathrm{SiO}_{2}$ film is used as an implantation cap (b). An n-well on photodetector is implanted by As ion at a dose of $1 \times 10^{15} \mathrm{~cm}^{-2}$ at $15 \mathrm{keV}$ to form a shallow junction ${ }^{(12)}(\mathrm{c}-\mathrm{d})$. The $\mathrm{p}+$ contact regions are formed by $\mathrm{BF}_{2}$ ion implantation at a dose of $2 \times 10^{15} \mathrm{~cm}^{-2}$ at 20 $\mathrm{keV}$ (e-g). A $50 \mathrm{~nm}$-thick $\mathrm{SiO}_{2}$ film is formed by plasma-enhanced chemical vapor deposition (PECVD) on the active region of the photodetector as a passivation for the sensing windows. The doped sample is annealed by RTA process at $1050^{\circ} \mathrm{C}$ for 10 seconds (h). The electrodes are formed by thermal evaporation of aluminum (i-j). Sintering process is introduced to form the ohmic contacts at $450^{\circ} \mathrm{C}$ for 15 minutes in $\mathrm{N}_{2}$ gas $(\mathrm{k})$. Next, a grating region is patterned on the active photodetector with a thick photoresist as a lift-off process. A $100 \mathrm{~nm}$-thick $\mathrm{Ta}_{2} \mathrm{O}_{5}$ film is deposited on the photodetector windows by RF-sputtering and the grating is formed by removing the photoresist for lift-off process (1). A $380 \mathrm{~nm}$-thick positive electron-beam (EB) resist (ZEP520A) is spin-coated on the device. A grating pattern is then transferred on the dielectric film by EB patterning (m). After developing, the grating is formed by fast atom beam (FAB) etching with $\mathrm{SF}_{6}$ gas, since the $\mathrm{FAB}$ has good directionality and anisotropical etching property (n).

\section{Results and Discussion}

Figure 7(a) shows optical micrograph of the fabricated photodetector. The scanning electron micrograph (SEM) of the cross-sectional view is also shown in fig. 7(b). The grating of the top silicon layer is fabricated well as shown in fig. 7(b). The measured thickness of the grating is $110 \mathrm{~nm}$ and the fill factor is 0.45 , which are $10 \%$ higher, $10 \%$ smaller than the designed values of $100 \mathrm{~nm}$ and 0.5 , respectively. As seen in fig. 7(a), the grating area covers $500 \times 650 \mu^{2}$ of the active $1000 \times 1500 \mu^{2}$ photodetector region. Due to the subwavelength grating period, a low reflection was achieved in the region.

I-V characteristics of the photodetector were measured without the resonant gratings. The measured characteristics are shown in fig. 8. The diode characteristics are clearly observed in the bias region from $-3 \mathrm{~V}$ to $0.5 \mathrm{~V}$. In spite of the thin silicon layer (380 $\mathrm{nm}$ ), a $p n$-junction is successfully formed vertically in the layer. The dark current was measured as $3.5 \mathrm{nA}$ at reverse bias voltage of $3 \mathrm{~V}$. The sensitivity was about $5 \mu \mathrm{A} / \mathrm{W}$ which is much smaller than the commercial silicon photodetectors because the sufficient depletion layer was not formed in the thin silicon layer, and also because only a small fraction of the incident light $(\sim 4 \%)$ was absorbed.

As depicted in fig. 9, the directional sensitivities were measured at the reverse bias voltage of $3 \mathrm{~V}$ for the $500 \times 650 \mu \mathrm{m}^{2}$ active resonant grating area with the periods of $450 \mathrm{~nm}$ and $350 \mathrm{~nm}$ at the wavelength of $783 \mathrm{~nm}, 6 \mathrm{~mW}$ incident light under TE polarization. We confirmed that the photocurrent was enhanced by the increased absorption at the resonat condition. The photocurrents measured as a function of the incident angle clearly show the resonant responses at the angle of 0 degrees and 25 degrees for the $450 \mathrm{~nm}$ and $350 \mathrm{~nm}$ periods, respectively. The directional angle for $350 \mathrm{~nm}$ period grating was about 25 degrees, which was smaller than that calculated ( 30 degrees) because of the fabrication errors. The directional sensitivity can be tuned by the change of the grating period. In the case of $450 \mathrm{~nm}$ grating period, the resonant response is obtained at the angle of 0 degrees, which

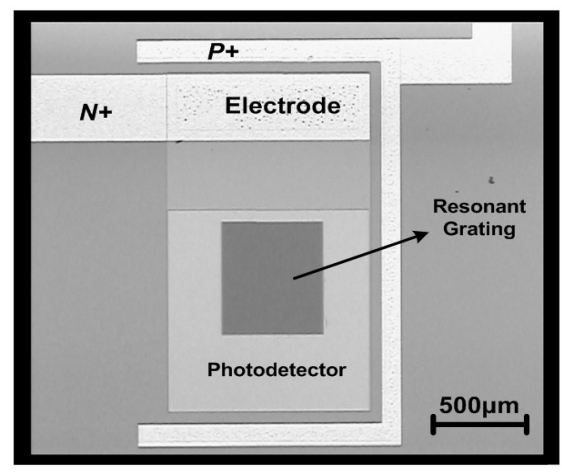

(a)

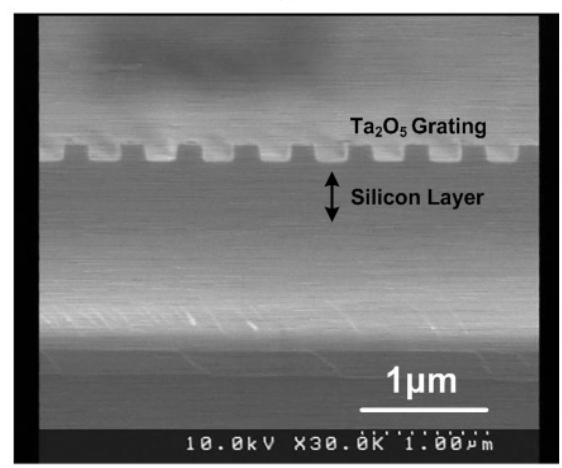

(b)

Fig. 7. Micrographs of fabricated directional thin-film photodetector. (a) top-view under optical microscope (b) cross-sectional view under SEM.

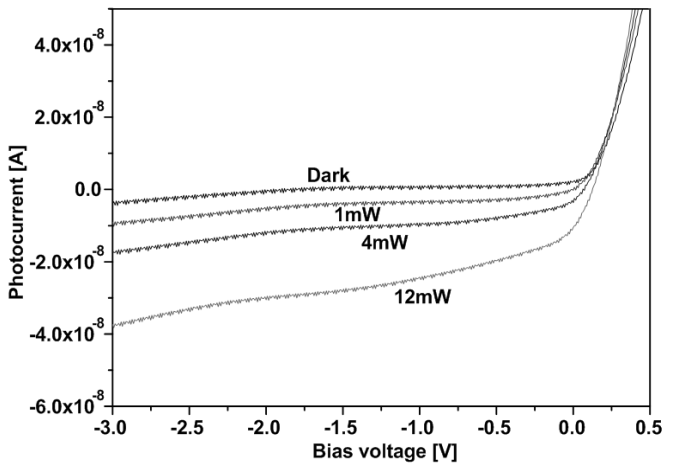

Fig. 8. I-V characteristics of the fabricated photodetector without resonant grating.

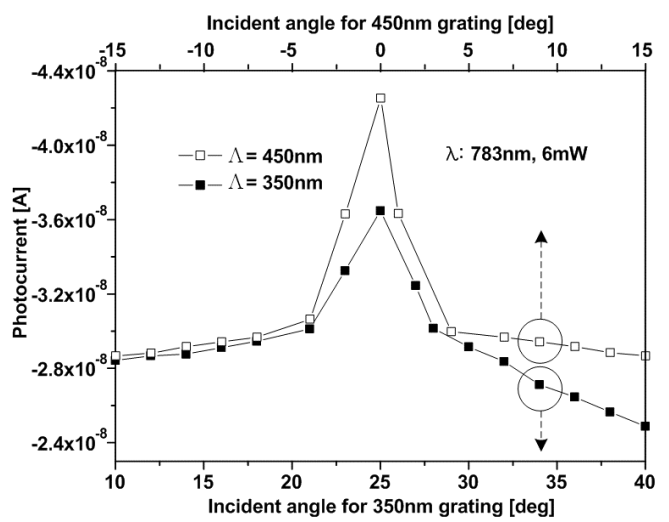

Fig. 9. Measured directional responsivity in the fabricated thin-film photodetector for $350 \mathrm{~nm}$ and 450 $\mathrm{nm}$ period gratings. 
agrees well with that obtained in the theoretical calculation. The enhanced photocurrents are less than the values expected from the absorbance shown in fig. 3. It can be considered the indistinct relations between the absorbed light and the photocurrents from the photoelectric effects, and imperfect boundary conditions or light scattering in the fabricated grating and layers are significant limiting the quantitative comparison between the measurement and calculation. However, the resonant phenomena in the thin silicon layer have been confirmed as shown in fig. 9 .

\section{Conclusions}

High functional photodetector is a key component for the optical precision measurement in the opto-mechatronic systems. To realize a compact and functional photosensor for an optical encoder, we proposed a directional thin-film photodetector. The device was designed using an optical simulation technique based on rigorous electromagnetic theory. A thin-film photodetector with angular sensitivity was fabricated based on silicon micromachining and conventional CMOS processes. In the measurements, the enhanced photocurrent was obtained by the guided mode resonant effect. Also the angular sensitivity of the fabricated sensor was confirmed.

(Manuscript received Oct. 13, 2006, revised Jan. 19, 2007)

\section{References}

(1) K. Hane, T. Endo, Y. Ito, and M. Sasaki : "A compact optical encoder with micromachined photodetector”, J. Opt. A: Pure Appl. Opt, Vol.3, pp.191-195 (2001-5)

(2) A. Teimel : "Technology and applications of grating interferometers in high-precision measurement", Precision Engineering, Vol.14, pp.147-154, (1992-7)

(3) P. Arguel et al. : "A monolithic optical phase-shift detector on silicon", IEEE Sensors J., Vol.5, pp.1305-1309 (2005-12)

(4) M. Sasaki, X. Mi, and K. Hane : "Standing wave detection and interferometer application using a photodiode thinner than optical wavelength", Appl. Phys. Lett., Vol.75, pp.2008-2010 (1999-10)

(5) M. Y. Liu, E. Chen, and S. Y. Chou : "140-GHz metal-semiconductor-metal photodetectors on silicon-on-insulator substrate with a scaled active layer", Appl. Phys. Lett., Vol.65, pp.887-888 (1994-8)

(6) S. M. Csutak, S. Dakshina-Murthy, and Joe C. Campbell : "CMOScompatible planar silicon waveguide-grating-coupler photodetectors fabricated on silicon-on-insulator(SOI) substrates", IEEE J. Quant. Electron., Vol.38, pp.477-479 (2002-5)

(7) H. R. Stuart and D. G. Hall : "Absorption enhancement in silicon-on-insulator waveguides using metal island films", Appl. Phys. Lett., Vol.69, pp.2327-2329 (1996-10)

(8) T. Ishi, J. Fujikata, K. Makita, T. Baba, and K. Ohashi : "Si-nano-photodiode with a surface plasmon antenna", J. J. Appl. Phys., Vol.44, pp.L364-L366 (2005-3)
(9) P. Cheben et al. : "Broad-band waveguide grating coupler with a subwavelength grating mirror", IEEE Photon. Technol. Lett., Vol.18, pp.13-15 (2006-1)

(10) T. Katchalskia, S. Soriab, E. Teitelbauma, A. A. Friesema, and G. Marowskyc : "Two photon fluorescence sensors based on resonant grating waveguide structures", Sensors and Actuators B, Vol.107, pp.121-125 (2005-5)

(11) P. S. Priambodo and T. A. Maldonado : "Fabrication and characterization of high-quality waveguide-mode optical filters", Appl. Phys. Lett., Vol.83, pp.3248-3250 (2003-10)

(12) A. A. Naem and I. D. Calder : "Formation of shallow $n+p$ junctions", J. Appl. Phys., Vol.62, pp.569-575 (1987-7)

Bongseok Choi (Non-member) received M.S. degree in

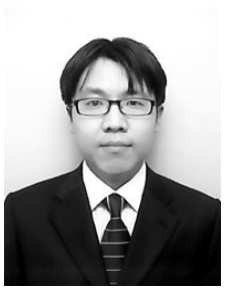
Mechatronics Engineering from Pukyong National University, Busan, Korea in 2004. He is currently a Ph.D. course student at the Department of Nanomechanics, Tohoku University. He is currently working on optical micro-sensors using MEMS technology.

Yoshiaki Kanamori (Member) received the MS and doctorate degree

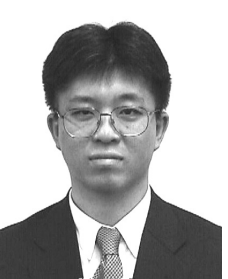
in engineering from Tohoku University in 1998 and in 2001, respectively. From 1998 to 2001, he was a Research Fellow of the Japan Society for the Promotion of Science. Since 2001, he has been a Research Associate of Graduate School of Engineering, Tohoku University. From 2003 to 2004, he was a postdoctoral researcher of Laboratory for Photonics and Nanostructures (LPN/CNRS) in France. He is currently engaged in the research and development of optical micro-sensors and optical MEMS.

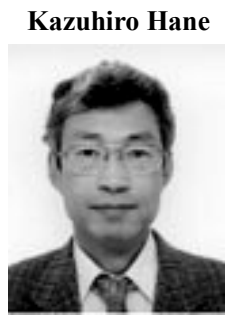

(Member) received the M.S. and Dr. Eng. Degrees from Nagoya University in 1980 and 1983, respectively. From 1983 to 1994 , he worked as a member of the Department of Electrical Engineering, Nagoya University. From 1985 to 1986, he was a guest researcher of National Research Council of Canada. Since 1994, he has been a professor of the Graduate School of Mechanical Engineering, Tohoku University, and is currently engaged in the research and development of microsensors and opto-mechanical system. He received the Numata Memorial Award and the Development Award of Japan Society for Precision Engineering in 1994 and 1996, respectively. 